\section{A case report of Multi System Atrophy (MSA) with cross over features of Progressive Supranuclear Palsy (PSP)}

\author{
Judah Rajendran $\mathbf{R}^{1 *}$, Ramesh Kannan² and Hannah Ranjee \\ Prasanth $^{3}$ \\ 1'Department of Neurology, Pondicherry Institute of Medical Sciences, Puducherry, India \\ 2Professor and HOD, Department of Neurology, Pondicherry Institute of Medical Sciences, \\ Puducherry, India \\ ${ }^{3}$ Professor, Department of Ophthalmology, Pondicherry Institute of Medical Sciences, Puducherry, \\ India
}

\section{Introduction}

We describe an interesting case of Multi System Atrophy who had cross over features of progressive supranuclear palsy along with classical clinical findings which led to the diagnosis.

Multi System Atrophy is a rare neurodegenerative, synucleinopathic disorder which progresses rapidly. It often presents with symptoms similar to Parkinsonism posing a diagnostic dilemma. It is an orphan disease with an annual incidence of 0.1 to 3 per 100,000, with many being revealed only on autopsy, following years of treatment for Parkinson's disease. There are very few cases reported from India, possibly because diagnosis requires a high index of suspicion, coupled with a comprehensive knowledge of the disease in order to detect it early and prevent progression.

MSA may involve the extrapyramidal system, pyramidal system, autonomic nerves and cerebellum. Clinical manifestations are Parkinson's symptoms, cerebellar ataxia, pyramidal tract signs and autonomic nervous system disorders. Based on the initial predominant motor deficits, MSA is subclassified as either Parkinsonian type (MSA-P) or cerebellar type (MSA-C). Progressive supranuclear palsy is an extremely rare presentation of MSA although it is sometimes difficult to differentiate the two. PSP is characterized by progressive supranuclear ophthalmoplegia, gait disorders, postural instability, dysarthria, dysphagia, muscle rigidity, and psychomotor slowing which is characterized by forgetfulness. PSP is also known as Steele- Richardson-Olszewski syndrome.

\section{Case report}

A 63 years old gentleman, farmer by occupation was admitted in the neurology ward with the chief complaints
More Information

*Address for Correspondence:

Dr. Judah Rajendran R, No: 22, 5th Main Street, Lakshmipuram, Thiruninravur, Chennai-602024, Tamil Nadu, India, Tel: +91-9003774686; Email: judah226@gmail.com

Submitted: August 23, 2021

Approved: September 10, 2021

Published: September 13, 2021

How to cite this article: Judah Rajendran R, Kannan R, Prasanth HR. A case report of Multi System Atrophy (MSA) with cross over features of Progressive Supranuclear Palsy (PSP). Int J Clin Exp Ophthalmol. 2021; 5: 019-022.

DOI: 10.29328/journal.ijceo.1001038

ORCiD: orcid.org/0000-0002-4342-6090

Copyright: @ 2021 Judah Rajendran R, et al. This is an open access article distributed under the Creative Commons Attribution License, which permits unrestricted use, distribution, and reproduction in any medium, provided the original work is properly cited.

Keywords: Progressive supranuclear palsy; Parkinson's disease; Diplopia; Vertical gaze palsy; Multisystem atrophy

(D) Check for updates

(d) OPEn ACCESS

of difficulty in speech for 18 months and blurred (defective) vision for 1 year. He had noticed a tendency to fall backwards over the last six months. He also complained of forgetfulness and fatigue which were gradually increasing over the last six months, for which he was on vitamin supplements, prescribed by a local doctor. He had history of trauma two years prior when he sustained injury to his left shoulder during a road traffic accident. There is no history of dysphagia/dyspnoea/ voice change/urogenital dysfunction/sleep disorders.

On general examination, he was conscious, oriented, obeying commands with stable vital signs. His GCS score was 15/15. Heart, lungs and abdomen were normal. Detailed CNS examination revealed that pupils were equal in size, $3 \mathrm{~mm}$, reacting briskly to light. Snout and glabellar reflexes were positive and cog wheeling was present. He had exaggerated deep tendon reflexes with an extensor plantar response and Romberg's sign was positive. He was not on any levodopa/ dopamine treatment.

Due to the defective vision, he underwent a thorough ocular examination and his visual acuity on Right eye was 6/12 and with pinhole $6 / 9$, visual acuity on Left eye was 6/9 and NIP. His colour vision was normal and there was no diplopia. Photomotor reflex and near convergence was normal. He was 
noted to have $15^{\prime}$ alternating exotropia with bilateral vertical gaze palsy in the upward direction. He had early cataract and Schirmer's test revealed a mild dryness of both eyes.

A differential diagnosis of Parkinson's disease, Multi System Atrophy, Progressive Supranuclear Palsy, Lewy body dementia and Corticobasal degeneration were considered, he was subjected to various investigations in order to confirm the diagnosis. EEG study was normal. A carotid duplex study revealed mild diffuse atherosclerotic wall changes. The MRI Brain showed age related atrophic changes in both cerebral hemispheres.

He was found to have neurogenic stuttering for which speech therapy was initiated. During his hospital stay, physiotherapy was given along with medications (Antiepileptics and Vitamin-E) and he improved symptomatically. His diplopia lessened and speech improved. He did not have any falls during his hospital stay. Since he showed considerable improvement, he was discharged and advised to continue his antiepileptics and vitamins.

\section{Discussion}

We report this case because of the rarity of multi system atrophy and the unusual presentation of supranuclear palsy. Our patient had been suffering with these symptoms for more than one and a half years, yet required only basic treatment to cause sufficient improvement in symptoms for him to enjoy a better quality of life.

It is difficult to differentiate PSP from MSA as there are so many similar features. Both may present with Parkinson's like symptoms and frequent history of falls. A few studies have noted that PSP patients present with oculomotor palsy, this is absent in MSA patients who are more likely to present with dysautonomia.

Multiple system atrophy (MSA) is the most rapidly progressive neurodegenerative disorder among the various types of synucleinopathies. The exact aetiology of MSA remains unknown, one postulated mechanism involves prion-like spreading of aberrant alpha-synuclein from neurons to glia through functionally connected networks, thereby leading to glial and myelin dysfunction and an inflammatory cascade that promotes secondary neurodegeneration. There have been a number of experimental studies demonstrating that abnormal alpha-synuclein aggregates might be responsible for the progression of MSA. However, this remains controversial and further studies are required to substantiate this theory [1].

The presence of alpha-synuclein messenger RNA (mRNA) in oligodendrocytes as well as glial inclusions and myelin disruption in the central nervous system suggests that MSA is a primary disorder of the glia. The glial cytoplasmic inclusions that are characteristic of MSA contain hyperphosphorylated alpha-synuclein, tau, ubiquitin, leucine-rich repeat serine/ threonine-protein kinase 2, and many other proteins [2].
Alpha-synuclein immunostaining is a sensitive marker of inclusion pathology in MSA. Neuronal inclusions of various types, including Lewy body-like inclusions, also are present in the majority of patients with MSA. Myelin degeneration is characteristic of MSA. A small case-control study found a significantly greater degree of white matter hyperintensities on magnetic resonance imaging (MRI) scans from patients with MSA compared with scans from patients with Parkinson disease and healthy controls [3].

Typical sites of pathologic involvement include the putamen, caudate nucleus, substantia nigra, pontine nuclei, inferior olivary nucleus, Purkinje cell layer of the cerebellum, and intermediolateral cell columns.

Autonomic dysfunction is secondary to loss of cells in the intermediolateral cell columns and loss of catecholaminergic neurons of the C1 area of the ventrolateral medulla (VLM). This is manifested by severe variability in blood pressure and heart rate, orthostatic hypotension, syncope, and postprandial hypotension [4].

In idiopathic Parkinson disease, positron emission tomography (PET) imaging studies have indicated that dysautonomia is caused by peripheral nervous system dysfunction, particularly myocardial sympathetic denervation. Bladder abnormalities in MSA include urinary incontinence and retention.

Motor abnormalities seen in MSA with predominant Parkinsonism (MSA-P) are due primarily to neuronal loss and gliosis in the substantia nigra, putamen, caudate, and globus pallidus. One of the features that distinguishes MSA and other atypical parkinsonian syndromes (Parkinson-plus syndromes) from idiopathic Parkinson disease is the lack of dramatic and sustained response to levodopa [5]. The extent of putamen involvement may determine the poor response to levodopa.

In contrast to MSA-P, the cerebellar ataxia and pyramidal signs that characterize the MSA with predominant cerebellar ataxia (MSA-C) subtype are secondary to degeneration of the cerebellar Purkinje cells, middle cerebellar peduncles, inferior olivary nuclei, and pontine nuclei. However, a majority of patients with MSA-P probably have subclinical loss of nigral neurons based upon findings from 123I-FP-CIT single-photon emission computed tomography (SPECT) imaging [6].

The role of genetics in the pathogenesis of MSA is undefined and some studies found mutations involve COQ2 gene, loss of the SHC2 gene and with genetic variants of the synuclein alpha $(S N C A)$ gene. But later study revealed the microtubuleassociated protein tau (MAPT) was the only gene that appeared to be associated with MSA, and two other studies suggest that MAPT haplotypes $\mathrm{H} 1 \mathrm{x}$ and $\mathrm{H} 1$ may be associated with an increased risk of MSA [7]. The role of tau isoforms in MSA is currently unknown. Genetic variants in the leucine- 
rich repeat kinase 2 (LRRK2) gene and the glucocerebrosidase $(G B A)$ gene, both common genetic causes of Parkinson disease, can present as MSA clinically as well as neuropathologically. Another study identified a new coiled-coil-helix-coiled-coilhelix domain containing 2 (CHCHD2) mutation in a patient with MSA [8-19].

MSA, previously called Shy-Drager syndrome, olivopontocerebellar atrophy or striatonigral degeneration were discontinued in1996, and the revised terminology has been in use since 2007.

Progressive Supranuclear Palsy (PSP) is a rare geriatric pathology, due to the abnormal deposition of tau protein, combining the motor tremor and bradykinesia of Parkinson's disease with the cognitive defects of Alzheimer's disease.

It has both sporadic and genetic role. It is found that MAPT mutations leads to inherited phenocopies of sporadic PSP. Other additional genes with increased risk are STX6, EIF2AK3 and MOBP with unclear significance. There is an increased risk of PSP with MAPT H1 haplotype with several subtypes (H1d, H1g and H1o) in which tau protein is abundant in the brains of PSP subjects [20-22].

PSP is the most common degenerative form of atypical Parkinsonism. The mean age of onset for PSP is approximately 65 years. No cases of PSP have been reported in patients younger than age 40 years. With the most common "classic" phenotype of PSP, known as Richardson syndrome (PSP$\mathrm{RS}$ ), the most frequent initial feature is a disturbance of gait resulting in falls.

Supranuclear ophthalmoparesis or ophthalmoplegia is the hallmark of PSP but may come on later. An important earlier manifestation is slowing of vertical saccades. Dysarthria, dysphagia, rigidity, frontal cognitive abnormalities, and sleep disturbances are additional common clinical features. PSP with predominant Parkinsonism (PSP-P) is characterized by asymmetric onset of limb symptoms, tremor, and a moderate initial therapeutic response to levodopa. It may be confused with idiopathic Parkinson disease.

The radiologic hummingbird sign (also called the penguin silhouette sign) results from the prominent midbrain atrophy in PSP with a relatively preserved pons, resembling a hummingbird or penguin in silhouette on midsagittal MRI of the brain [7].

The most consistent sites of microscopic findings in PSP are in the basal ganglia, particularly in the substantia nigra, subthalamic nucleus, and internal globus pallidus, in addition to the oculomotor complex, periaqueductal gray matter, superior colliculi, and dentate nucleus. Involvement of the cerebral cortex is also increasingly recognized. The histologic characteristics of PSP include neuronal loss, gliosis, and the presence of tau-positive filamentous inclusions in specific anatomic areas involving astrocytes (most notably the hallmark finding of tufted astrocytes), oligodendrocytes, and neurons. Tau cytoplasmic inclusions in surviving neurons, known as globose neurofibrillary tangles, are classically described in PSP, but are not specific to PSP [4].

The diagnosis of PSP during life is based upon the clinical features. Suspicion for PSP is raised when new-onset neurologic, cognitive, or behavioural deficits progress in absence of other identifiable cause in a patient $\geq 40$ years of age. The core clinical features include postural instability, oculomotor deficits, especially vertical gaze palsy, akinesia/ parkinsonism, and frontal lobe impairments, including speech and language problems and behavioural change. No laboratory or imaging studies are diagnostic. Neuropathologic examination remains the gold standard for its definitive diagnosis.

The disorders that are the most difficult to differentiate from PSP are the other neurodegenerative parkinsonian disorders such as idiopathic Parkinson disease, corticobasal degeneration, multiple system atrophy, and dementia with Lewy bodies, as well as vascular parkinsonism.

Parkinson's disease being a close differential diagnosis leads to the prescription of dopamine unnecessarily which has its own side effects like Angina pectoris, atrial fibrillation, bradycardia, ectopic beats, hypertension, hypotension, palpitations, tachycardia, vasoconstriction, ventricular arrhythmia, ventricular conduction, widened QRS complex on ECG, anxiety, headache, Gangrene (high dose), piloerection, Azotaemia, Increased intraocular pressure, mydriasis and tissue necrosis. Thus proper evaluation and management is needed for a better outcome.

\section{Conclusion}

Multi System Atrophy is a rare disorder, which may present with atypical features. It is an important differential diagnosis for Parkinson's disease and should be recognized early. Physicians and ophthalmologists should be acquainted to this disease and its treatment strategies in order to slow progression and ensure optimal quality of life. A multidisciplinary approach, as was taken with our patient, is vital to ensure encouraging results. Further studies will be helpful in elucidating the precise pathogenesis and effective treatment. Attention to the few characteristic features will lead physicians to the diagnosis and improve subsequent management.

\section{References}

1. Respondek G, Levin J, Höglinger GU. Progressive supranuclear palsy and multiple system atrophy: clinicopathological concepts and therapeutic challenges. Curr Opin Neurol. 2018; 31: 448-454. PubMed: https://pubmed.ncbi.nlm.nih.gov/29746401/

2. Schrag A, Ben-Shlomo Y, Quinn NP. Prevalence of progressive supranuclear palsy and multiple system atrophy: a cross-sectional study. Lancet. 1999 20; 354: 1771-1775.

PubMed: https://pubmed.ncbi.nlm.nih.gov/10577638/ 
3. Bower JH, Maraganore DM, McDonnell SK, Rocca WA. Incidence of progressive supranuclear palsy and multiple system atrophy in Olmsted County, Minnesota, 1976 to 1990. Neurology. 1997; 49: 1284-1288. PubMed: https://pubmed.ncbi.nlm.nih.gov/9371909/

4. Steele JC, Richardson JC, Olszewski J. Progressive supranuclear palsy. A heterogeneous degeneration involving the brain stem, basal ganglia and cerebellum with vertical gaze and pseudobulbar palsy, nuchal dystonia and dementia. Arch Neurol. 1964; 10: 333-359. PubMed: https://pubmed.ncbi.nlm.nih.gov/14107684/

5. Joutsa J, Gardberg M, Röyttä M, Kaasinen V. Diagnostic accuracy of Parkinsonism syndromes by general neurologists. Parkinsonism Relat Disord. 2014; 20: 840-844.

PubMed: https://pubmed.ncbi.nlm.nih.gov/24816002/

6. Cosseddu M, Benussi A, Gazzina S, Manes MA, Dell'Era V, et al Natural history and predictors of survival in progressive supranuclear palsy. J Neurol Sci. 2017; 382: 105-107.

PubMed: https://pubmed.ncbi.nlm.nih.gov/29111000/

7. Glasmacher SA, Leigh PN, Saha RA. Predictors of survival in progressive supranuclear palsy and multiple system atrophy: a systematic review and meta-analysis. J Neurol Neurosurg Psychiatry. 2017; 88: 402-411. PubMed: https://pubmed.ncbi.nlm.nih.gov/28250027/

8. Schrag A, Ben-Shlomo Y, Quinn NP. Prevalence of progressive supranuclear palsy and multiple system atrophy: a cross-sectional study. Lancet. 1999; 354: 1771-1775.

PubMed: https://pubmed.ncbi.nlm.nih.gov/10577638/

9. Takanashi M, Ohta S, Matsuoka S, Mori H, Mizuno Y. Mixed multiple system atrophy and progressive supranuclear palsy: a clinical and pathologicalreportofonecase. ActaNeuropathol(Berl).2020;103:82-87. PubMed: https://pubmed.ncbi.nlm.nih.gov/11837750/

10. Gearing M, Olson DA, Watts RL, Mirra SS. Progressive supranuclear palsy: neuropathologic and clinical heterogeneity. Neurology 1994; 44 1015-1024.

PubMed: https://pubmed.ncbi.nlm.nih.gov/8208392/

11. Dickson DW, Lin WL, Liu WK, Yen SH. Multiple system atrophy: a sporadic synucleinopathy. Brain Pathol. 1999; 9: 721-732.

PubMed: https://pubmed.ncbi.nlm.nih.gov/10517510/

12. Bower JH, Maraganore DM, McDonnell SK, Rocca WA. Incidence of progressive supranuclear palsy and multiple system atrophy in Olmsted County, Minnesota, 1976-1990. Neurology. 1997; 49: 1284-1288. PubMed: https://pubmed.ncbi.nlm.nih.gov/9371909/
13. Winter $Y$, Spottke AE, Stamelou M, et al. Health-related quality of life in multiple system atrophy and progressive supranuclear palsy. Neurodegener Dis. 2011; 8: 438-446.

PubMed: https://pubmed.ncbi.nlm.nih.gov/21576919/

14. Glasmacher SA, Leigh PN, Saha RA. Predictors of survival in progressive supranuclear palsy and multiple system atrophy: a systematic review and meta-analysis. J Neurol Neurosurg Psychiatry. 2017; 88: 402-411. PubMed: https://pubmed.ncbi.nlm.nih.gov/28250027/

15. Multiple System Atrophy Research Collaboration. Mutations in COQ2 in familial and sporadic multiple system atrophy. N Engl J Med. 2013; 369: 233-234.

PubMed: https://pubmed.ncbi.nlm.nih.gov/23758206/

16. Scholz SW, Houlden H, Schulte C, Sharma M, Li A, et al. SNCA variants are associated with increased risk for multiple system atrophy. Ann Neurol. 2009; 65: 610-614.

PubMed: https://pubmed.ncbi.nlm.nih.gov/19475667/

17. Sailor A, Schlz SW, Nalls MA, Schulte C, Federoff M, et al. A genomewide association study in multiple system atrophy. Neurology. 2016; 87: 1591-1598.

PubMed: https://pubmed.ncbi.nlm.nih.gov/27629089/

18. Vilarino-GuellC,Soto-OrtolazaAI,RajputA,MashDC,PapapetropoulosS, et al. MAPT $\mathrm{H} 1$ haplotype is a risk factor for essential tremor and multiple system atrophy. Neurology. 2011; 76: 670-672.

PubMed: https://pubmed.ncbi.nlm.nih.gov/21321341/

19. Labbe C, Heckman MG, Lorenzo-Betancor O, Murray ME, Ogaki K, et al. MAPT haplotype diversity in multiple system atrophy, Parkinsonism Relat Disord. 2016; 30: 40-45.

PubMed: https://pubmed.ncbi.nlm.nih.gov/27374978/

20. Donker Kaat L, Boon AJ, Azmani A, Kamphorst W, Breteler MMB, et al. Familial aggregation of parkinsonism in progessive Supranuclear Palsy. Neurology. 2009; 73: 98-105.

PubMed: https://pubmed.ncbi.nlm.nih.gov/19458322/

21. Heckman MG, Brennan RR, Labbe C, Soto Al, Koga S, et al. Association of MAPT subhaplotypes with Risk of Progressive Supranuclear Palsy and Severity of Tau Pathology. JAMA Neurol. 2019; 76: 710-717. PubMed: https://pubmed.ncbi.nlm.nih.gov/30882841/

22. Im SY, Kim YE, Kim YJ. Genetics of Progressive Supranuclear Palsy. J Mov Disord. 2015; 8: 122-129.

PubMed: https://pubmed.ncbi.nlm.nih.gov/26413239/ 